\title{
دور المكتبة الجامعية في بيئة هجتمع المعلومات: دراسة نظرية
}

\section{أحمد حافظا إبراهيهم \\ كلية الآداب / الجامعة المستنـصرية

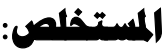

هدفت الدراسة إلى التعرف على مفهوم مجتمع المعلومات و الدور الجديد للمكتبات الجامعية في ظل مجتمع

المعلومات ،تم اتباع منهج البحث العلمي الوثائقي لملاءمته لطبيعة البحث.توصلت الدراسات إلى العديد من النتائج منها تعد المكتبة الجامعية العقل النابض للجامعة لكونها تهتم بالعملية التعليمية وبنقل المعرفة للباحثين بمختلف تخصصـاتهم

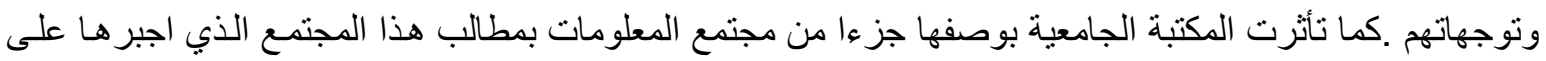

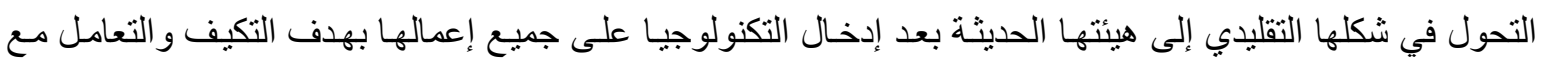
مجتمع المعلومات. إما أهم المقترحات التي جاءت بها الدر اسة فهي على المكتبات الجامعية تهيئة بنية تحتية تكنولوجية إعلية

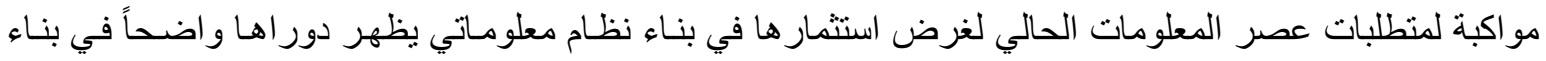
وتتمية المجتمع و إعادة هيكلة الإمكانيات المادية و البشرية داخل المكتبـة والسعي إلى تطبيق معايير الجودة في إتاحسة المعلومات الالكترونية المتوفرة فيها والاستفادة من التقنيات الحديثة وضرورة دعمها مادياً من قبل الجامعة.

\section{Abstract:}

The study aimed to identify the concept of the information society and the new role of university libraries in the light of the information society, has been to follow the scientific method documentary research for suitability to the nature of Seat.touselt studies a number of results, including the university library is the mind pulsating League for being interested in the educational process and the transfer of knowledge to researchers of various specializations and orientations. Also affected by the university Library as part of the information society demands of this society, which forced it to shift in its traditional form to the bench after the introduction of modern technology on all its work in order to adapt and deal with the information society The most important proposals that came in the study are the University Libraries Thaoh technological infrastructure keep pace with the requirements of the current era of information for the purpose of investing in the construction of an information system shows Douraha evident in building and community development and restructuring of material and human resources inside the library and seek to implement quality in the availability of electronic information available standards and the use of modern technologies and the need to financially supported by the university.

\section{الاقدمة:}

تعد المكتبات الجامعية اسبق من غير ها من أنواع المكتبـات الأخرى في استخدام التكنولوجيـا الحديثة، وخدمات البحث الإلية والمعالجة الإلية للمعلومـات نظر اً لدور ها الهام في مساندة التعليم والبحث العلمي في لهي الجامعة، وقد انعكس ذلك على الخدمات التي تقدمها ، فهي تعد أكثر المكتبـات اهتمامـاً وتطوير اً للخدمات البحثية التي أصبحت تعد أكثر من ضدرورية للباحثين ولضمان بقائها وتمكينها من مواجهة التحديات التي البحث العلمي في ظل التطورات التقنية. حيث إن الغاية الحقيقية لوجود المكتبات الجامعية هي تقديم الخدمات لمختلف فئات الباحثين و أعضاء هيئة التدريس وطلبة الدر اسات العليا سواء داخل وخار ج الجامعة، فضلاً عن لن 
طلبة المرحلة الجامعية الأولية، وتتضح أهمية المكتبات الجامعية في كونها تتيح للباحث الاطلاع على احدث

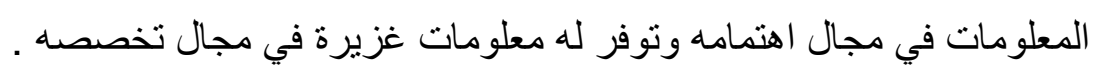

تعـد المكتبـات الجامعيـة مـن المؤسسـات التي تـدل على تطـور وتقدم الأمسم وتتحـد مشـلة البحـث

بالتساؤ لات التالية:

ا - ما هو الدور الجديد للمكتبات الجامعية في ظل التطورات الجديدة لمجتمع المعلومات؟ r- ما أثر التكنولوجيا الحديثة على أداء المكتبات الجامعية و على مستوى الخدمات التي تقدمها لمختلف فئات

\section{أهداف البمث:

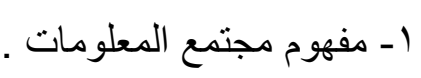

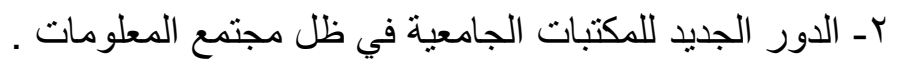
r- التحديات التي تواجه المكتبات الجامعية في عصر المعلومات.

تم استخدام المنهج الوثائقي والاطلاع على البحوث و المقالات العلمية ذات العلاقة بموضوع البحث.

\section{فرضيات البمث:}

ا ـ استخدام التكنولوجيـا الحديثـة يسـاعد على تحسين أداء المكتبـات الجامعيـة وتوصيل باحثيهـا إلى مـوارد المعلومات. rـ لمجتمـع المعلومـات اثر في تغيـر طبيعـة خدمات المكتبـة الجامعيـة وسـلوك باحثيهـا في بحثهم عـن المعلومات.

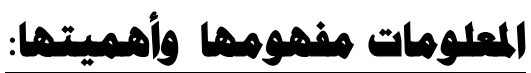

تعد المعلومات من أهم مقومات الحياة ومن أبرز ركائز التقدم الحضـاري ، ولها ارتباط وثيق بجميع

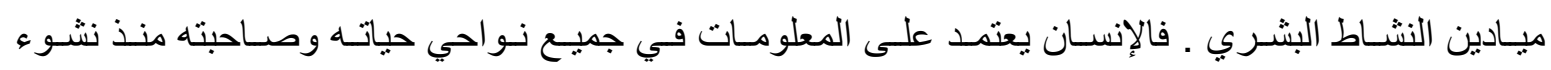
المجتمعـات البشـرية، وفي هـذا العصـر ظهـر اهتمـام متز ايــ بالمعلومـات كونهـا ثـروة وطنيـة تلعـب دورًا

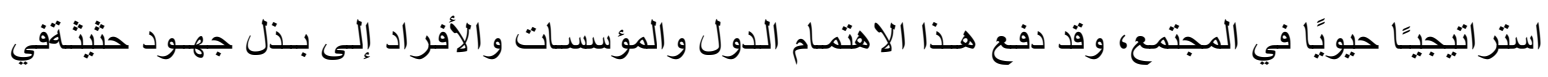

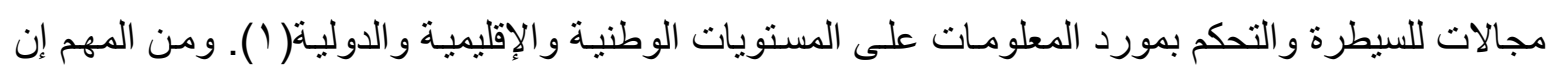
نشير إلى إن للمعلومات جانبان هامان هما: اـ الجانب الذهني : وهو الجانب الفلسفي أي بمعنى إن المعلومات محتويات العلاقة بين أنثياء ماديـة متداخلة ومتفاعلة مع بعضها تبرز نفسها في حالة تغير من هذه الأشياء. r- الجاتب الوثائقي: حيث تستعمل المعلومات في هذا الجانب للدلالتة على الوثائق أو غيرهـا من المسجلات

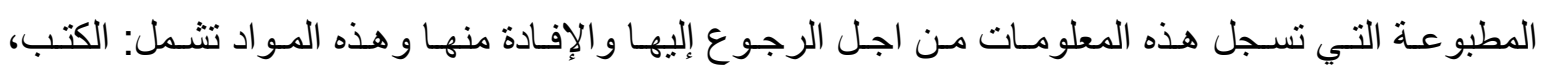


النشـرات، الـدوريات، إضـافة إلـى المـواد السـمعية والبصـرية ومــا متــاح على شبكة الانترنـت مـن بيانـات و ومعلومات هائلة الكترونية، رقمية، افتر اضية و غير ها(Y).وتعتبر عملية تلبية حاجات المعلومات عملية معقدة وتحتاج إلى فهم عميق لطبيعة هذه الاحتياجات ، لأنها تعبر عن سبب استخدامها وكيفيته اتخاذ القرار على ونى

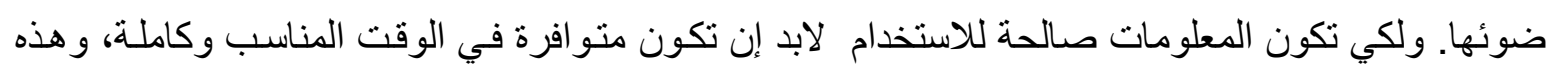
هي الجودة النوعية للمعلومات(ب).

\section{أهمية المالومات:}

للمعلومات أهمية كبيرة حيث دخلت كل تفاصيل الحياة الإنسانية ولها دور هـا في تتميـة قدرات الفرد و المجتمع وتطور الأنشطة وفعاليات الحياة المختلفة كما إن لها الدور الأساس في عملية البحث العلمي فلولاهـا لإهـا

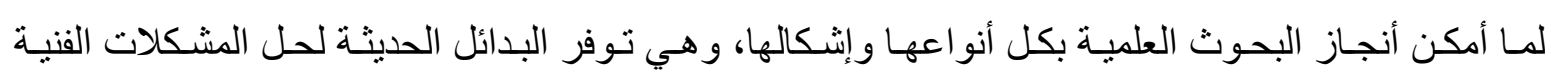
وتساعد على اتخاذ القرارات السليمة في جميع القطاعات. وتمكن الدولة من رسم سياستها العامة. و هناك من يلخص أهمية المعلومات بالنقاط التالية(؟):

ا ـ تعد العنصر الأساسي في اتخاذ القرار المناسب وحل المشكلات. r- لها دور كبير في إثراء البحث العلمي وتطور العلوم والتكنولوجيا. بـ ـا لها أهمية كبيرة في مجالات التنمية الاقتصادية و الاجتماعية والإدارية و الثقافية و الصحية. ع ـ تساهم في بناء استر اتيجيات المعلومات على المستوى الوطني و والعالمي.

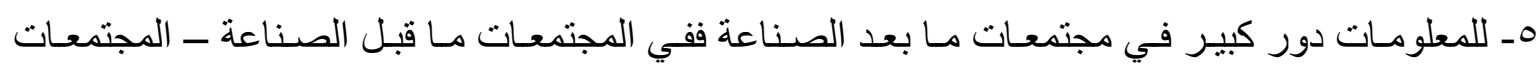
الزر اعيةـ كان الاعتماد على المواد الأولية والطاقة الطبيعية كالرياح و الماء و الحيوانات والجهد البشري، إمـا

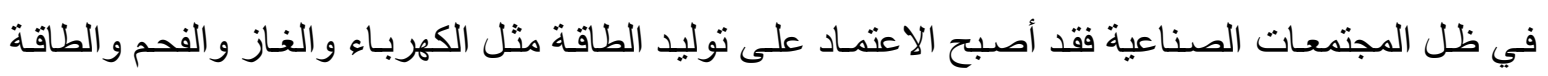
النووية. اـ المعلومـات تسـاعد في نقل الخبر ات للآخرين و على حل المشكلات التي تواجهمه و على الاستفادة من المعرفة المتاحة.

\section{مشكلات المصول على المعلوهات:}

بالرغم من الأهمية القصوى للمعلومات إلا إن هنالك بعض المشكلات قد تقف عائقاً في الحصول عليها

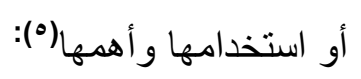

ا ـ ظهور المعلومات بلغة واحدة وقد تكون هذه اللغة غير معروفة عالميـاً مما يؤدي إلى صعوبة الاستفادة منها ז- لا يمكن الاعتماد على الدوريات التي تتشر المستخلصات لأنها لا تغطي البحوث و الدراسـات الهامـة التي

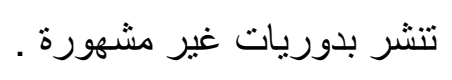

r- قصور الخدمات المكتبية التقليدية عن تلبية احتياجات المستفيدين المختلفة . ع - المعلومات ذاتها لا يمكن السيطرة عليها لسر عة صدور ها وتداخلها وتطور ها وتتوع أثنكالها وصور ها. 


\section{هجتمع المعلومهات}

يمتاز مجتمع المعلومات بأنه يركز على العمليات التي تعالج فيها المعلومات لتصبح المعرفة هي المـادة الخام الأساسية، وبالتالي فأن المعرفة تؤدي إلى توليد معرفة جديدة عكس المواد الأساسية التي سرعان مـان تتضب في المجتمعات الأخرى بسبب الاستهلاك ، إما في مجتمع المعلومات فالمعلومـات تولد المعلومـات( (7). إما مجتمع المعلومات فهو ذلك المجتمع الذي يتعامل مع المعلومات بأسلوب مستمر ومتطور وفعال، تستخدم فيه تكنولوجيا المعلومات والاتصالات عند بعد ومـا يرتبط بهما من تطورات اجتماعية و اقتصسادية وسياسية

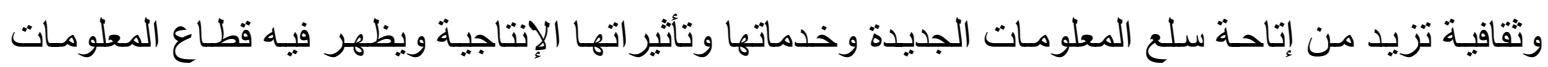

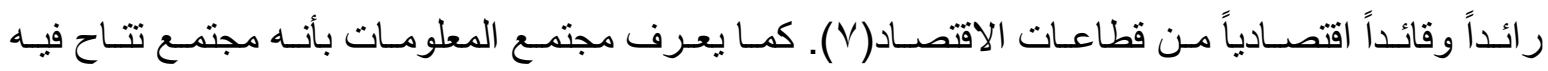
الاتصالات العالمية وتنتج فيه المعلومات بكميات ضخمة ، موز عة توزيعاً واسعاً ، وتصبح فيه المعلومات قوة

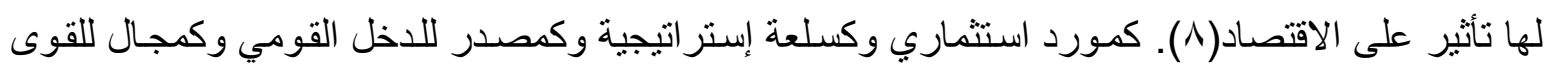
العاملة) (9).

وقد استعرضت سهير عبد الباسط خصائص مجتمع المعلومات ، كالتالي:- ( ( )

منظمات كثيفة المعلومات.

$$
\text { قطاع معلومات فعال. }
$$

الاستخدام الاجتماعي للمعلومات ،إي استخدام المعلومات بصورة كبيرة بين الجمهور العام.

$$
\text { وجود تعلم مدى الحياة. }
$$

تحول قوة العمل من أنتاج السـلع والخدمات الماديـة وتوزيعها إلى إنتاج سـع المعلومـات ومعالجتها

وتوزيعها.

توفير بنية أساسية قوية تتيح الوصول من اي مكان الى المصادر الغنية بالمعلومات. توصيل الخدمات على الخط المباثر. التجارة الالكترونية. تعدد فئات المتعاملين مع المعلومات.

المكانة الخاصة للمعرفة العملية. اهتمام الحكومات بقضايا مجتمع المعلومات. الاهتمام بالسياسات الخاصة بتكنولوجيا المعلومات و الاتصالات. تنامي تكنولوجيا المعلومات.

\section{الاستراتيجيات المستفدهة من قبل الكتبيين والاختصاصيين في هجتمع المعلوهات هي: (II)}

هناك خطوات معينة يجب العمل بها وصو لاً إلى الاستفادة القصوى من المعلومات المتاحة هي:

$$
\text { ا ـ العمل على توحيد المنتجين المزودين لمصادر المعلومات المتتوعة. }
$$
r- استخدام التوجيهات و الإرشادات الداعية إلى أهمية العمل الجماعي و التعاوني. 


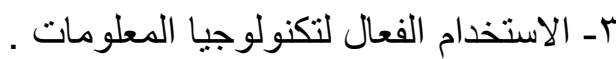

ع- مساعدة الناس على تعلم الوصول إلى المعلومات من خلال استغلال مصادر مجتمع المعلومات. هـالتغلب على العوائق الجغر افية والتحديات الأخرى من خلال التطوير الفعال للاتصالات عبر الحدود. 7- تقوية العلاقة ما بين مزودي المعلومات و المستخدمين. V-تبني اتجاهات مبادرة . 1-المشاركة في تغطية النفقات.

ويمكن للبنيـة الأساسية للمعلومـات على الصـيدين الـوطني والعـالمي، في مجتمعــا المعاصر ، إن تشتملما يلي:

1 -شبكات الاتصالات بعيدة المدى المرتبطة ببعضها البعض ، القابلة للتشـيل التبادلي على نحو يكفل تجنب الانقطاع. r- نظم الحاسبات، وأجهزة التلفزة، والاتصالات الهاتفية، والفاكس. rـ البرمجيات وخدمات المعلومات ، ومر اصد البيانات ، ومر افق المعلومات ، بما في ذللك المكتبات.

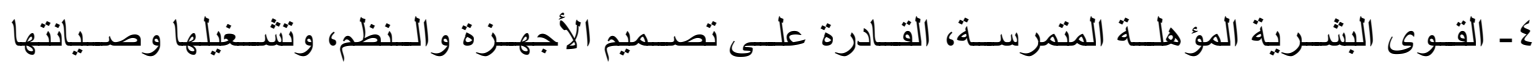
وتطوير ها(Y ( ).

وعموماً فان مؤشرات مجتمع المعلومات تتظور على امتداد أربع مراحل مترابطة: 1 -الجاهزية: و هي ترتبط بالبنى الأساسية الفنية والتكنولوجية والاجتماعية. rـ الكثافـة: و هـي تبـرز حالــة اسـتخدام تطبيقـات تكنولوجيـا المعلومـات و الاتصــالات فـي اي مجتمـع مـن المجتمعات. rـ الأثر : ويقصد به النتائج التي تترتب على استخدام تكنولوجيا والاتصالات.

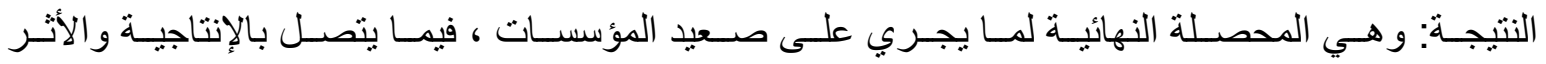
الاجتماعي( M (1).و عموماً ترجع أصول مجتمع المعلومات إلى تطورين مرتبطين بعضهما البعض همـا:التطور الاقتصادي طويل الأجل. و التغير التكنولوجي( ع اعنع ().

ويرى بعض الباحثين إن إطار مجتمع المطلومات يتركز في الملامح التالية:

ا ـالمنفعة المعلوماتية من خلال إنشاء بنية تحتية معلوماتية على أساس الحاسبات العامـة المتاحة لكل النسا، في صورة شبكات المعلومات المختلفة وبنوك المعلومات ، التي ستصبح هي بذاتها رمز المجتمع. rـ الصناعة الر ائدة ستكون هي صناعة المعلومات التي ستهيمن على البناء الصناعي . r- سيتحول النظام السياسي لكي تسوده الديمقراطية التشـاركية، وتعني السياسـات التي تنهض على لـى أسـاس الإدارة الذاتية التي يقوم بها المواطنين. ع- سيتشكل البناء الاجتماعي من مجتمعات محلية متعددة المر اكز ومتكاملة بطريقة طوعية . 
هـ ستتغير القيم الإنسـانية وتتحول من التركيز على الاستهلاك المـادي إلى إثباع الانجـاز المتعلق بتحقيق الأهداف.

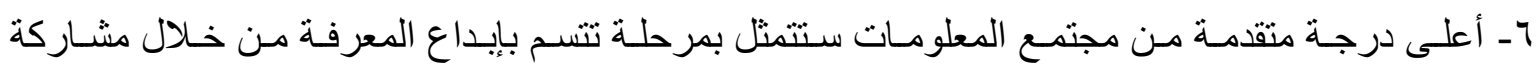

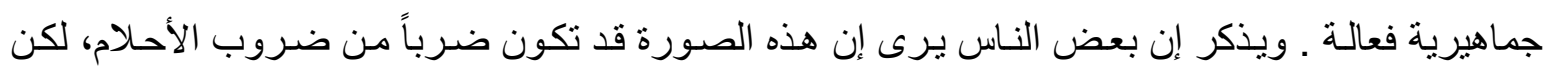
هناك بعض الباحثين يقولون إن هنالك أدلة إننا نتحدث عن و اقع وليس عن حلم من الأحلام (0 ( ).

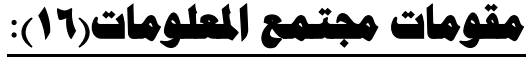

يستند مجتمع المعلومات على بعض المقومات الهامة منها:

سياسة وطنية للمعلومات و المعرفة ودعم السلطة العليا في الدولة بشكل مستمر وقوي.

أنظمـة وتشـريعات ملائمسة مثنل حقوق الملكيـة الفكريـة وبـر اءات الاقتـر اع وحمايـة البيانـات وحريـة

التعبير وحرية النفاذ إلى المعلومات.

بيئة تعليم وبحث و إبداع وتطوير مناسبة تودي إلى كو ادر وطنية مؤهلة.

آليات التمويل الملائمة .

الوعي ألمعلوماتي او ثقافة المعلومات و انتشار ها في المجتمع.

وهناك سمات يجب أن تتوفر في المجتمع لكي يمكن إن يوصف بأنه مجتمع معلومات أهمها(V V):

1- إن تتوفر فيه تكنولوجيا جيدة للمعلومات تمزح بين أنتاج وحفظ وتداول المعلومات و التقنيات الحديثة . r - نظام جديد لمؤسسات المجتمع يستخدم النظام الثبكي المفتوح ويسهم بالمعلومات. بـ الانتقال من المعلومات إلى المعرفة.

ع- ظهور نظام اقتصادي جديد يعبر الحدود الدولية (الثركات متعددة الجنسيات أو عابرة الحدود). 0ـ - مظام سياسي عالمي.

ويستخلص وليم مارتن مجموعة من المعايير التي يمكن من خلالها الحكم على تحول المجتمع إلى

مرحلة مجتمع المعلومات وهي(1 1 ):

1- المعيار التكنولوجي: عندما تصبح تكنولوجيا المعلومات والاتصال مصدر القوى الأساسية في المجتمع. r- المعيار الاقتصادي: عندما تبرز المعلومات و المعارف كمورد اقتصـادي مهم وكمصدر للقيمة المضافة.

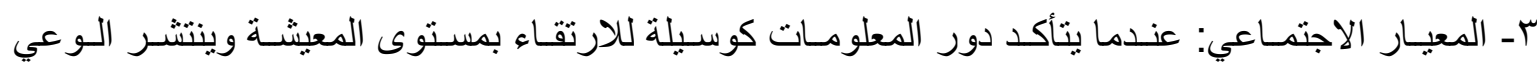
بالمعلومات.

ع- المعيار السياسي: عندما تؤدي حرية المعلومات إلى تطوير العملية السياسية وزيادة مشاركة الجماهير في اتخاذ القرار ات بطريقة رشيدة مبنية على استعمال المعلومات.

هـ المعيار الثقافي: عندما يتأكد الاعتر اف بـالقيم الثقافيـة للمعلومـات كاحتر ام حقوق الملكية الفكريـة و الصدق الإعلامي و الأمانة العلمية وترويج هذه القيم من اجل الصالح القومي. 


\section{و هناك ثلاث خصائص رئيسية أساسية تتحكم في مجتمع المعلومات (9 (1):}

الخاصية الأولى هي: استخدام المعلومات كمورد اقتصادي حيث تعمل المؤسسـات و الثشركات على

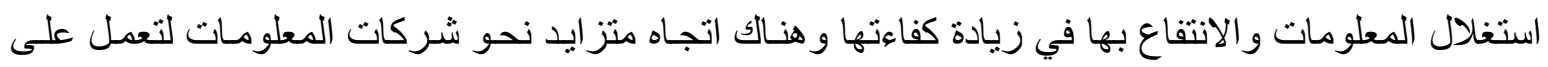

$$
\text { تحسين الاقتصاد الكلي للدولة }
$$

الخاصية الثانية: هي الاستخدام المتناهي للمعلومات بين الجمهور العام

الخاصية الثالثة: هي ظهور قطاع المعلومات ، كقطاع مهم من قطاعات الاقتصاد.

ولكي تدخل المكتبـة الجامعية مجتمع المعلومـات يرى الباحث انه لابـ عليها إن تعمل على إعداد كو ادر ها وتدريهم لكي يكونو ا مؤهلين على التعامل مع تكنولوجيا المعلومات الحديثة والتطور ات المستقبلية .

\section{الاكتبة الجامعية الرفية والتطوير(·):}

لابد في البداية من إعطاء تعريف شامل للمكتبة فالمكتبة هي القلب النابض لأي مؤسسة، حيث يبدأ فيها

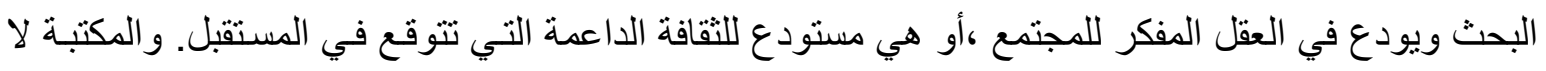

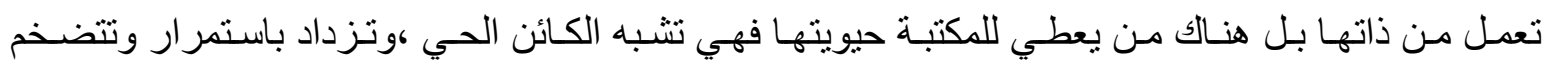

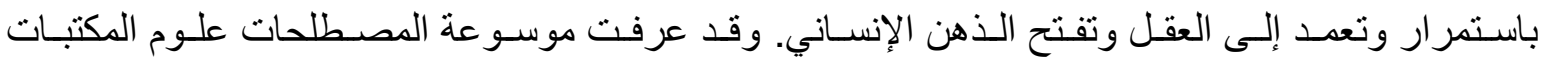

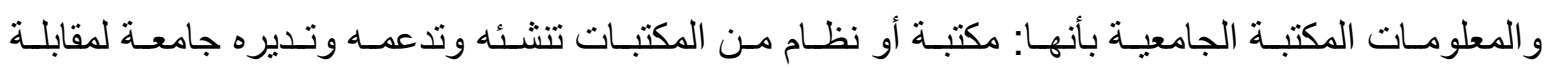

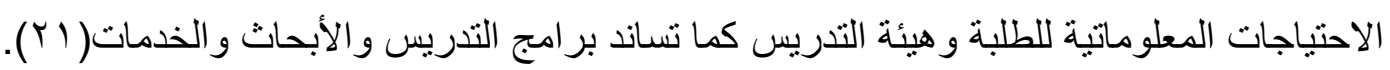
ويطلق أيضا على المكتبات الجامعية المكتبات الأكاديمية وتعرف بأنها تللك المكتبات الموجودة في

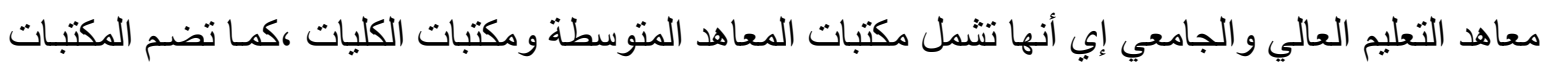

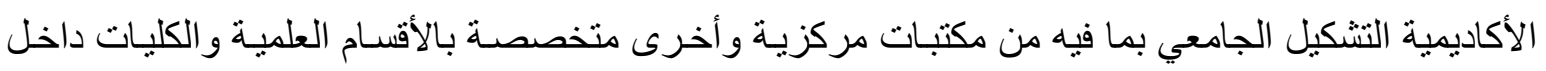

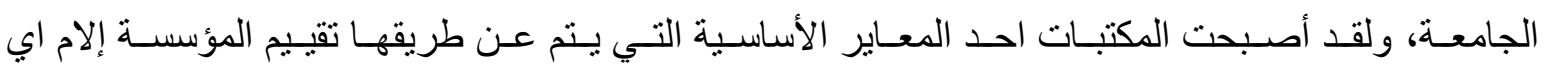

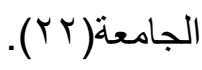

و على الرغم تعدد تعريفات المكتبة الجامعية، إلا أنها في مجمو عها تتفق على إن المكتبة الجامعية

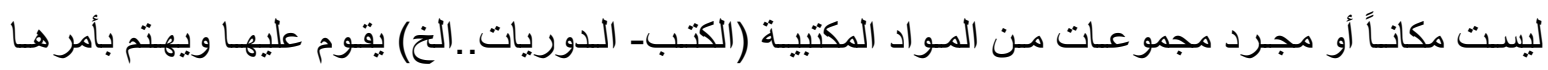

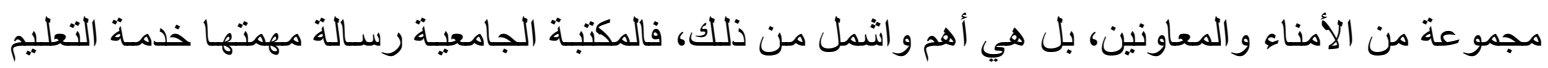

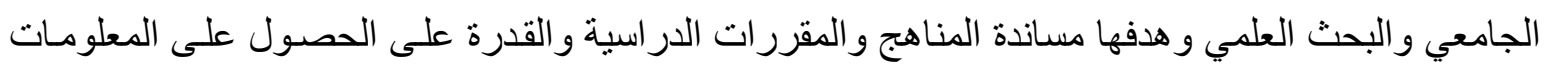

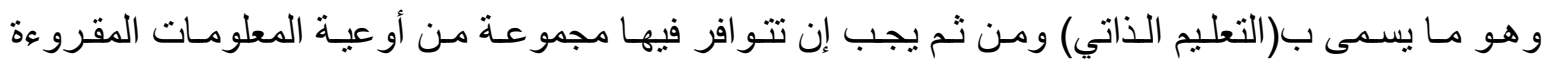

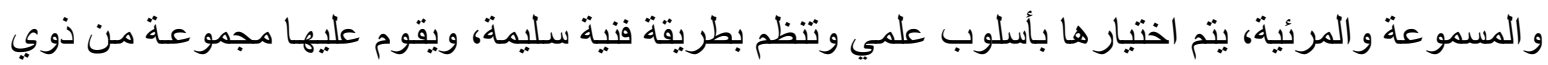

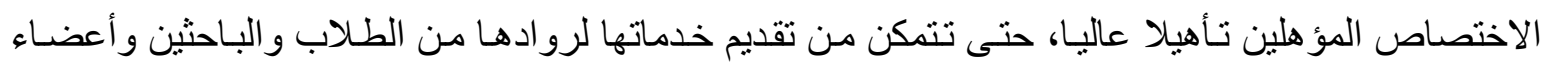

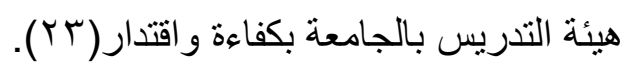

إذن فالمكتبة الجامعية هي مؤسسة ثقافية وتثقيفية وتربوية وعلمية تعمل على خدمة مجتمع معين من

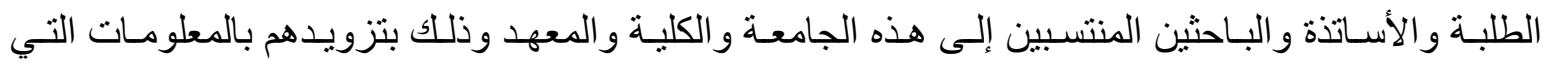


يحتاجونها في دراستهم و أبحاثهم من الكتب والدوريات والمر اجع و أوعيـة المعلومـات الأخرى بعد تنظيمها

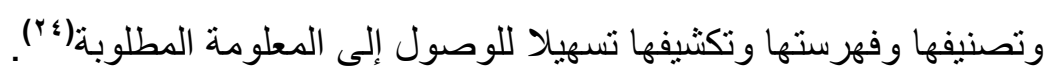

تقوم المكتبـة الجامعيـة بـدور اً محوريـاً وأساسيا على مسـتوى الجامعـة مـن خـلال النهوض بـالتعليم

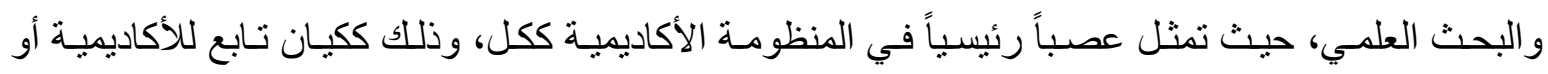

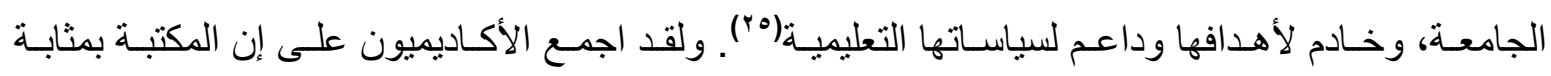

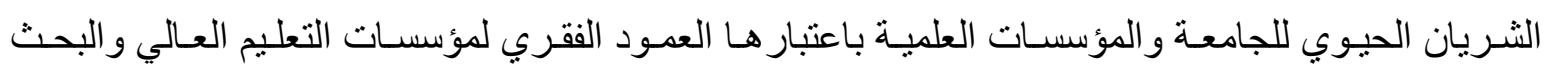

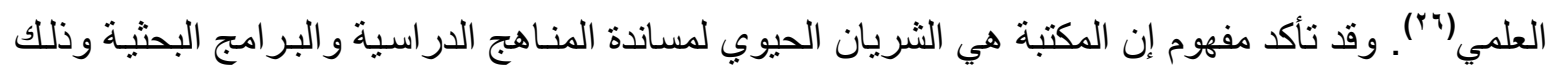

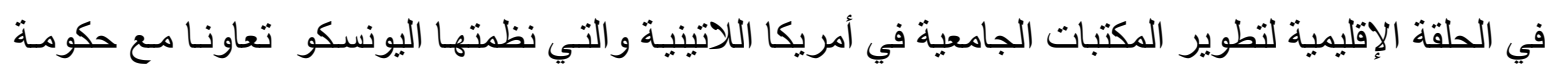
الأرجنتين حول دور المكتبة في حياة الجامعة ، حيث خلصت الحلقة إلى ما يلي(YV) : - - إن مستوى رقي أو تقدم الدولة يعتمد بدرجة كبيرة على مستوى التعليم العالي فيها. - إن مستوى التعليم العالي بعتمد بدرجة كبيرة على ما تضطلع به الجامعات. - - إن نجاح الجامعات مرتبط بصلاحية وكفاءة مكتباتها الجامعية.

إذن فالمكتبة الجامعية بالمفهوم العلمي الحديث هي إحدى المؤسسـات الثقافية التي تؤدي دوراً علميـاً أنياً

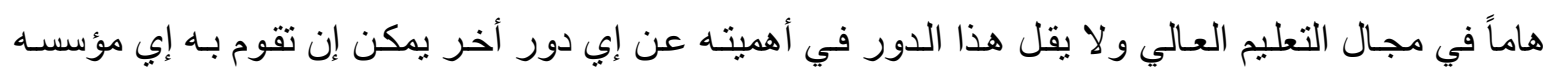

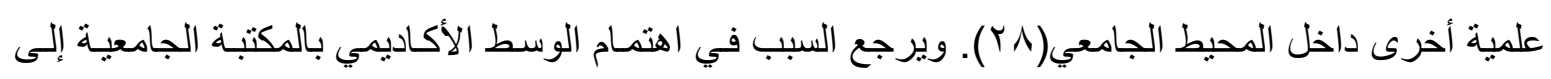
ما توفره من معلومات لأسرة الجامعة من أسـاتذة وطلبة وبـاحثين في شتى حقول المعرفة. ويتوقف نجاح المكتبة الجامعية على مدى قدرتها وفاعليتها في توفير خدمات معلومات رفيعة المستوى بحيث تلبي احتياجات المستفيدين في فترة زمنية وجيزة وبشئ من الثمولية. ولا يقتصر دور المكتبة على توفير احدث معلومـات فحسب، لكنه يشتمل على ما تقوم به في نشر الوعي المكتبي الذي يركز على تدريب المستفيدين كافة في كيفية

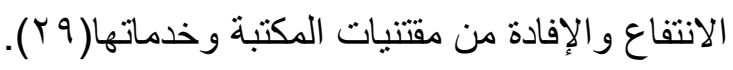

ويثير احمد بدر إلى إن المكتبة الجامعية يمكن إن تكون بذاتها مؤسسـة تعليميـة ، غير انسه يؤكد على أنها تكون أكثر كفاءة وفعالية و إنتاجية عندما تحاول إن تؤدي وظيفتها الرئيسية كأداة مكملة للعملية التعليميـة في الجامعة و عندما تكون جزء لا يتجزأ من المنهج الدراسي و التكويني على جميع المستويات التعليمية. حيث تؤدي المكتبة الجامعية رسالة متميزة تتمثل في استمر ارية التعليم كسبيل لتطوير المجتمع الأكاديمي ذلك لان وندي سرعة تغير المعلومـات و المعـارف وازديـاد حجمها وتخصصـها يقتضـي النظر إلى التعليم على انسه عمليـة مستمرة( (ب).

ويـرى الباحث إن الدور التربـوي والتعليمي للمكتبـات يتلخص في توفير مصسادر المعرفـة وتدعيم المناهج الدراسية وتوفير المعلومات وتدعيم الأنشطة البحثية وتتميـة عادة القراعة والاطلاع وتتميـة المهارات

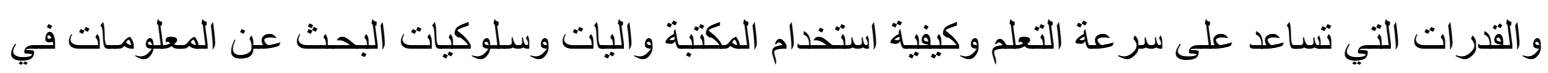
مختلف مجالات مصادر المعرفة المتاحة في المكتبة. 


\section{المكتبة الجامعية في عصر المعلومات والتكنولوجيا:}

وضعت تكنولوجيا المعلومات و الاتصـال الحديثة المكتبة الجامعية على طريق التكنولوجيا الحديثة وضـرورة توظيفها في العمل المكتبي ، ولم يعد إمامها بديل سوى دخول هذا المجتمع بوعي و إدر الك وفهم لطبيعـة هذا المجتمع ومن اجل إرساء قو اعدها في هذا المجتمع لابد للمكتبة الاهتمام بثلاث جوانب محورية هي: أو لاً: الهيكل الإداري والتنظيمي للمكتبة ثانياً: المستقيدين، ثالثاً: سلوك البحث عن المعلومات. حيث تغير مفهوم العمل في المكتبات الجامعية بحكم التطورات الحديثة في مجال خدمات المعلومـات و واسترجاعها و إتاحتها للمستفيدين، وأصبحت معطيات التكنولوجيا الحديثة بدائل ضرورية لرفع مستوى الأداء و الإنتاجية وظهور شبكات المعلومات نتيجة للتطورات التي حدثت في مجال التخاطب الالكتروني بين أجهزة الحاسبات مما سهل عملية تبادل ونقل المعلومـات بكافة أنو اعها و إثـالها . ممـا أدى إلى حدوث تغيير كبير على مهام ووظائف المكتبات ليصل إليها الباحثين بأسرع و أسهل الطرق( (ب).

و على الرغم من ذلك تواجه المكتبات في العصر التكنولوجي مجمو عة من التحديات أهمها(بr):

ا - ثورة المعلومات التي تفرز كل ساعة كميات هائلة من المعلومـات العلميـة بحيث لا يمكن لأي مكتبة في العالم إن تدعى لنفسها الاكتفاء الذاتي مهما بلغت إمكاناتها المادية ومواردها البشرية. r- تنوع إثكال مصادر المعلومات التقليدية و السمعية و البصرية و الالكترونية و غيرها. r- ت تنوع احتياجات المستفيدين ومطالبهم وعمق تخصصاتهم كماً وكيفاً.

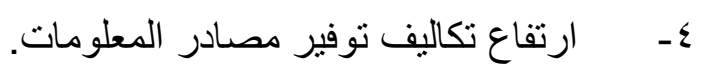

وقد أشارت المعايير الموحدة الأمريكية(rT) على ضرورة إن تكون مجموعات مكتبـة الجامعة ذات إحجام ونوعية ملائمة لسد كافة الاحتياجات التعليمية وتسهيل بر امج البحث الجامعية، فيجب على المكتبة إن تشتمل على المصـادر الضـرورية المتصـلة بالمنـاهج الدراسية أو التي تسـاعد البـاحثين في إعداد أبحـاثهم وتكـون مجموعـات المكتبـة على درجـة عاليـة من الجودة بحيث تسمح بالارتقاء بالبحث العلمي، وان تكون مناسبة لاحتياجات و اهتمامات أعضاء هيئة التدريس وطلبة الدر اسات العليا و الباحثين سواء داخل أو خـارج الجامعـة، فضلاً عن طلبة المرحلة الجامعية الأولية.

ويرى الباحث ض ضرورة محافظة المكتبـة الجامعيـة على تو افقها مـع التكنولوجيا السـائدة وان تستخدم

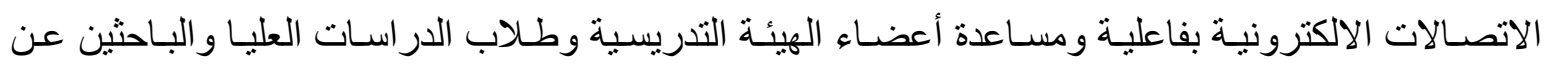
طريق توفير ها لمختلف مصـادر المعلومـات. وضرورة توجهها لمواجهة الانفجار المعرفي وتحقيق عنصر السيطرة على المعلومات من خلال التعاون فيما بينهما. سواء أكانت مكتبات الكليات في الجامعة الو احدة أو بين المكتبات الجامعية على المستوى المحلي أو العالمي من خلال ربط المكتبات والجامعات ببنوك المعلومـات وشبكات المعلومات العالمية والإفادة من أساليب الفهرسة التعاونية والأعمال و البيلوجر افية والخدمات الأخرى التي توفرها بعض النظم التعاونية. 


\section{العواهل التي أدت إلى استفدام التقنيات المديثة في المكتبات الجامعية هي( ع):}

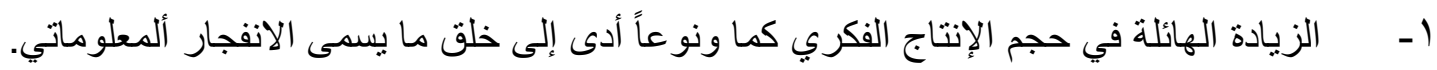

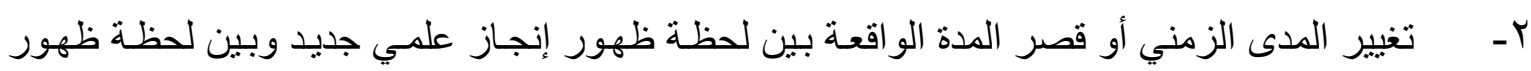
إنجاز علمي آخر.

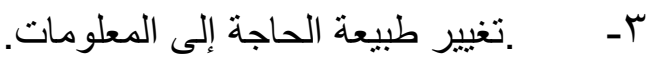
ع- تززايد الطلب على المعلومات ذات الصبغة العالمية نتيجة لازدياد عمليات التبادل الاقتصادي و الثقافي والاجتماعي.

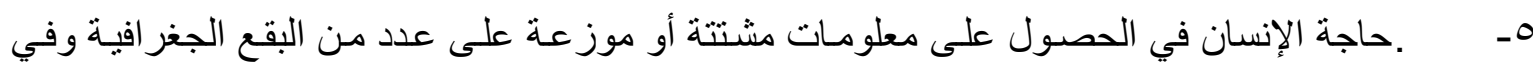
أماكن متفرقة وعجز الوسائل التقليدية عن تلبية هذه الاحتياجات. 7- تحقيق النعاون الإيجابي بين أمين المكتبة الجامعية وأعضاء الهيئة التنريسية.

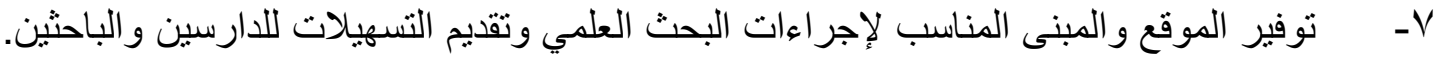
1- تقديم خدمات معلومات جديدة ومنطورة مثل الإحاطة الجارية والبث الانتقائي للمعلومات وإعداد و البلو غر افيات و المستخلصات والكثافات والاهتمام بتعزيز الخدمات الإعلامية مثل بر امج خدمات المكتبات الجامعية وكسب جمهور المستفيدين.

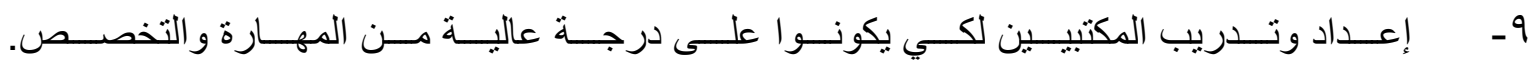

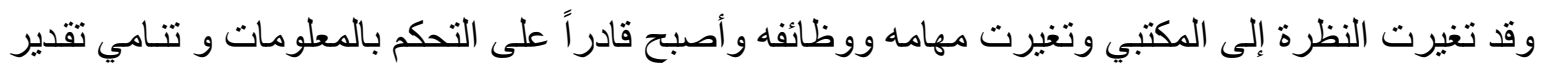
المجتمع لمتتهنين هذا العمل الذي أصبح يطلق عليه (أخصائي المعلومات).

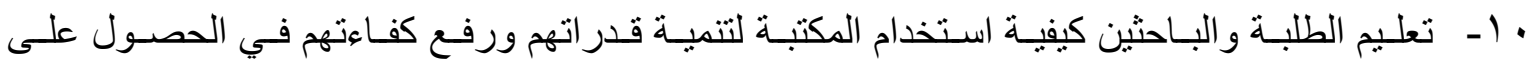
المعلومات وكذلك التركيز على خدمات الإرشاد والتوجيه.

ويرى الباحث ضرورة إنشاء قو اعد بيانات لإبراز النشاطات والإسهامات العلمية للباحثين وأعضاء

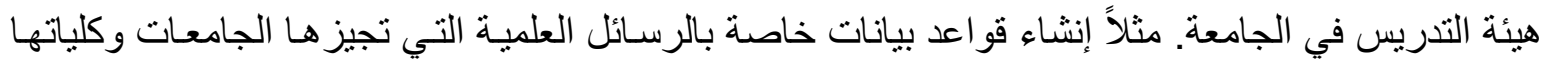
الدختلفة وقاعدة بيانات البحوث الجاريـة وقاعدة بيانات الكتب النادرة المتوفرة في الجامعة وقاعدة بيانات خاصة ببر اءة الاختراع لأساتذة الجامعة، و العمل على تجاوز الصعوبات الناجمة عن تتوع وتثتت المصادادر

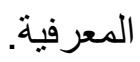

ويمكن توظيف واستخام التقتيات الحيثة في المكتبات الجامعية إن يحقق الأهداف التالية لغرض مواكبة مجتمع المطلومات (ro) (ro)

ا- القدرة على تحسين الفئات المستفيدة بديمومة الاتصـال(Permanent Connection ) ووجود متابعة مكتبية من اجل تبسيط نظام التحاور وزيادة فرص التجول المكتبي في قاعدة البيانات المتاحة.

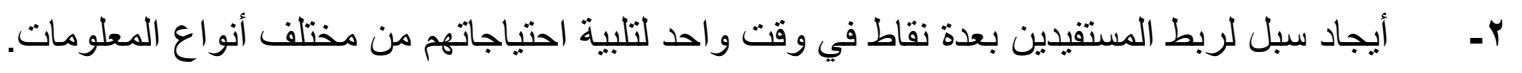




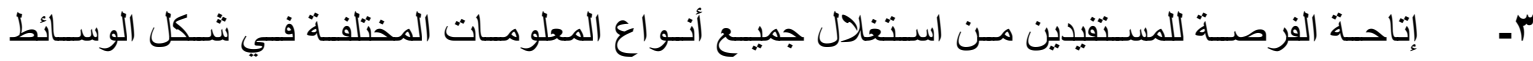
المتعددة(Multi Media Data Banks).

إما أهم مميزات استخدام تقتيات المعلومات والاتصالات في المكتبات الجامعية فهي كما يأتي(؟ب): 1- زيادة الفاعلية وتطوير الأداء في العمليات الفنية والخدمات.

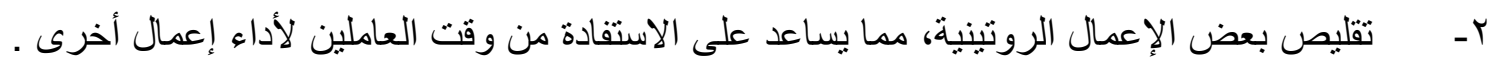

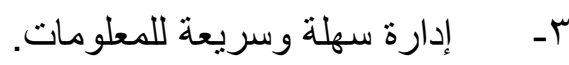
ع-التحسين في طرق و أساليب التعاون مع المستفيدين من المكتبة. 0- توفير طاقات خزن واسترجاع عالية للمعلومات و البيانات. 7- الرغبة في تقديم خدمات معلومات جديدة ومتطورة .

\section{الدور البدديد للامكتبة الجامعية في ظل مجتمع المعلومات}

ذكر بيتر سون إن اثثين من الأسباب الرئيسية التي أدت إلى تغير الدور التقليدي للمكتبات ،الأول هو التحول إلى التكنولوجيا الرقمية، والثاني هو إعداد الطلاب المتزايدة و الطموحة أكاديميـا التي يمكن تسميتها (بالجيل الرقمي) الذي يتوقع من المكتبة الجامعية إن تلبي احتياجاتهم وتساعدهم في التعلم بسر عة، وعليه فان التحدي الذي يجب إن نعرفه اليوم كأمنـاء مكتبـات يتمثنل في ضرورة تطوير هياكل و أسس تتظيمية وفنيـة جديدة لتقديم خدمـة مكتبيـة مزدوجـة تجمـع بين الوسط التقليدي و الوسط الالكترونـي بحيث تمكننـا فعـلا من هن الاندماج و التأقلم مع التغيرات المستمرة التي تحدث في عالم الوسائط المعلوماتية ، ومن ثم فـان المكتبـة عليها إن تجمع بين مفهومي المكتبـة التقليديـة والمكتبـة الاكترونية. وبالتالي ينبغي على المكتبـة الجامعية إن تتبنى استر اتيجيات تتناسب مع تطور ات القرن الحادي و العشرين وذلك من خلال أربع محاور أساسية وهي (rV):

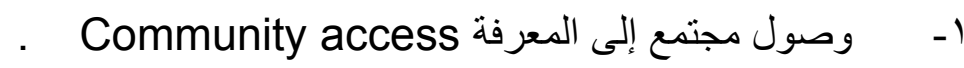

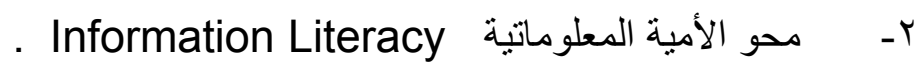

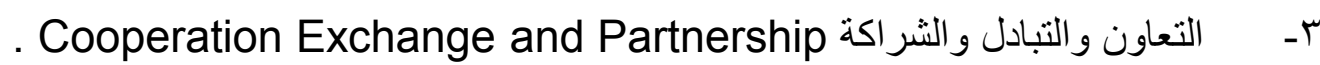

عـ - المعارض و الفعاليات العلمية Exhibitions and Scholarly Events.

و أصبح لزامـاً على المكتبـة الجامعيـة إن تقوم بوظيفة جهاز المعلومـات المتطور الذي يبقي من هذا الفيض ألمعلوماتي ما يخدم أعضاء هيئة التدريس و الباحثين والطلبة. فالمكتبة الجامعية في مجتمع المعلومـات أصبح لز امـاً عليها مو اكبـة ثورة المعلومـات والولوج إلى عـالم الأوعيـة الرقمية والنشـر الالكترونـي وتـامين

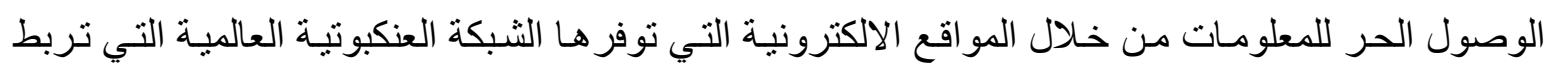
الجميع مهمـا كان تواجدهم في العـالم ، ونظر الموقعها في قمـة الهرم العلمي ومنـارة البحث الأكاديمي إذن عليها ان تسـاهم إيجابـا في تحقيق أهداف الجامعة العلميـة والتعليميـة باعتبار هـا احد عناصر تقيهم الجامعـات

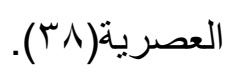




\section{ومن بين التحديات التي تواجه الجامعة ومكتباتها في ظل مجتمع المطومات هي ما ياتي(9 ؟):}

1 - التطور التكنولوجي: إن التطور الذي يشهده العالم اليوم ينبئ بان امتلاك هذه الأشياء وتكديسها يعد خطوة

نحو السيطرة على ماديات الكون والياته، لكن دون إن نغفل عن امتلاك الأفكار التي تتحكم في هذه الأتشياء. r- العولمة: إن المجتمعات الحديثة عبارة عن تجمعات بشرية تشكلت دون توحد المكان فالثبكات جعلت من

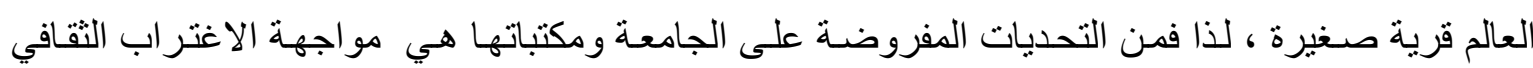
للأمة. و العمل على تماسك ووحدة الإفر اد و انسجامهم داخل كيانات اجتماعيـة متجانسـة تحقق التنوع و التقدم المنشود.

Г- الاتفجـار المعرفي: بعد إن أصبحت المعلومـات عبـارة عن ثنائيـة رياضية في وقتنـا الحاضـر، تغيرت استر اتيجية المكتبات بشكل جذري من خلال توفير ها لكم هائل من المعلومـات الرقميـة و الالكترونيـة مـع ترك الاختيار للمستفيد.

\section{عناصر نجاح المكتبة الجامعية :}

لكي تتمكن المكتبـة الجامعيـة من أداء وظيفتها وتحقيق أهدافها في خدمة التدريس والبحث لا بـ من عناصر أساسية في برنامج المكتبة الجامعية أهمها( • ع) :

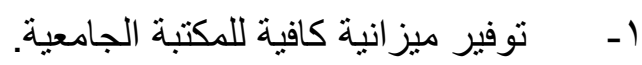

r- وجود مصادر المعلومات اللازمة للتعليم والبحث و الإرشاد و التوعية. ب- توفر الموارد البشرية الكفوءة. ع - تنظيم مو اد المكتبة للاستخدام. هـ تكامل مصادر المكتبة مع مصادر المعلومات في شبكة المكتبات الوطنية أو الدولية. 7- نو افر المباني والمساحة والتجهيزات التي تتلاعم مع استخدام المكتبة. V- تكامل سياسة المكتبة مع السياسات الإدارية و التعليمية للجامعة . م- وجود علاقة إدارية سليمة بين المكتبة والإدارة العليا للجامعة. 9- تشكيل أو تنظيم مكتبي يتفق مع ظروف الجامعة إلام. • 1 القيام بخدمات معلومات وخدمات مرجعية متميزة.

و تقترح كرستين بورجمان( ( ) نموذجاً للمهار ات و المعارف التي يحتاج إليها المكتبين أو المستقيدين

لمواجهة التحديات التي تواجه المكتبة في ظل عصر المعلومات منها :

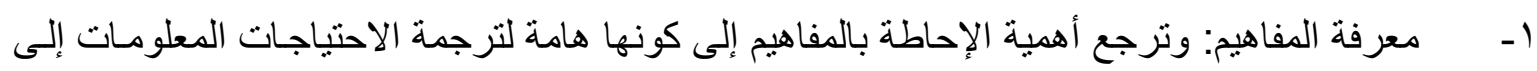
خطة ينفذ البحث عن المعلومات من خلالها. r- معرفة الدلالات و التر اكيب: يقصد بالمعرفة الدلالية الإحاطة بالعمليات المتاحة لتنفيذ خطة البحث، بينما تعني المعرفة التراكييية قدرة المستفيد على استيعاب الأوامر التي يستخدمها نظام معلومات بعينة.

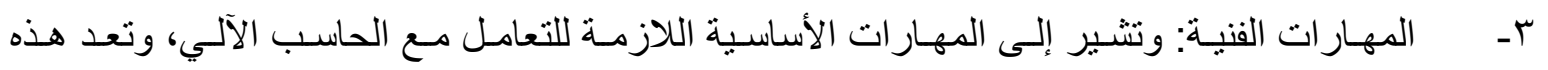
المهار ات ضرورية لتنمية كل من المعرفة التصورية والمعرفة الدلالية التر اكييية. 
و لاثـك في إن المكتبـة الجامعيـة التي لـديها أمنـاء مكتبـات على مسـتوى عـالٍ مـن الكفـاءة والتأهيـل و التدريب وتحمل المسئولية ستكون قادرة على تقديم خدمـة فعالـة للمستفيدين منها. بالإضـافة إلى مـا ير اه الباحث من إن على أمناء المكتبات و المعلومات إن يتحلنون بمجموعة من القدرات اهمها: 1 - القدرة على البحث في قو اعد البيانات ومو اقع الويب.

r- اختيار المجموعات الرقمية واقتنائها وتتظيمها و إدارتها. r- القدرة على حوسبة العمل داخل المكتبة الجامعية، تحويل المصادر المطبو عة للشكل الرقمي. ع- القدرة على الإبحار و التصفح على شبكة الانترنت . 0- - تحليل ومعالجة مختلف أنواع مصادر المعلومات. 7- امتلاك الوعي لتقديم خدمات المعلومات بفاعلية.

1 - تعد المكتبـة الجامعية العقل النـابض للجامعة لكونها تهتم بالعملية التعليميـة وبنقل وتوطين المعرفة للباحثين بمختلف تخصصاتهم وتوجهاتهم . r- تأثرت المكتبة الجامعية (باعتبار ها جز ء من مجتمع المعلومات) بمطالب هذا المجتمع التأثثرات التي نلاحظها الذي اجبر هـا التحول في شكلها التقليدي إلى هيئتها الحديثة بعد إدخـال التكنولوجيـا على جميع إعمالها بهدف التكيف و التعامل مع مجتمع المعلومات. r- للمكتبة الجامعية دور كبير في تطوير البحث العلمي في ظل عصر المعلومـات من خـلال مـا توفره من مصادر المعلومات و إتاحتها للباحثين. ع - هناك الكثير من التحديات ( الداخلية والخارجية) التي تواجه المكتبات الجامعية منها العولمة، و التقدم العلمي ، و الطفرة الهائلة في التقدم التكنولوجي وثورة الاتصالات. 0ـ يساعد استخدام التكنولوجيـا المعلوماتيـة الحديثة في المكتبـات الجامعيـة على تحسين الأداء و إمكانية

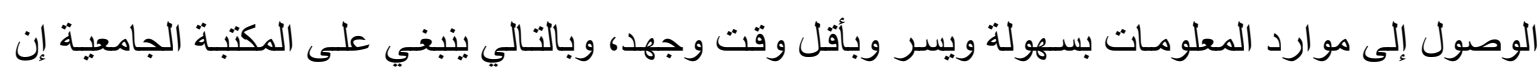
تتبنى استر اتيجيات تتناسب مع تطور ات استخدام التقنيات الحديثة. 7- ترتب على التقدم العلمي و استخدام التقنيات الحديثة العديد من التغير ات انعكست بدور ها على المكتبة

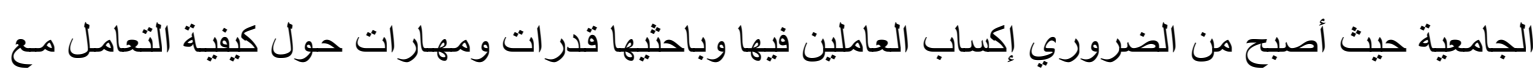

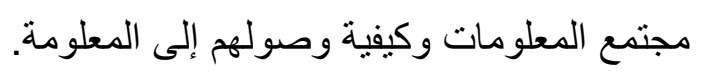

|المترحات: 1 - على المكتبـات الجامعيـة تهيئة بنيـة تحتيـة تكنولوجيـة مواكبـة لمتطلبـات عصـر المعلومـات الحسالي لغرض استثمار ها في بناء نظام معلوماتي يظهر دور ها واضحاً في بناء وتتمية المجتمع . r- إعادة هيكلة الإمكانيات المادية والبشرية داخل المكتبة و السعي إلى تطبيق معايير الجودة في إتاحسة

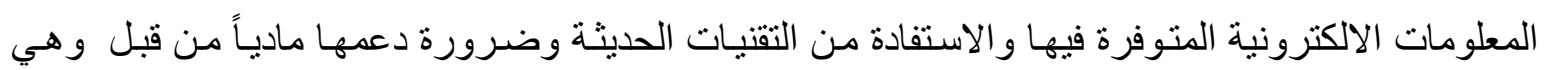
الجامعة. 
r- - إتاحة الفرصة للعاملين في المكتبة الجامعية للالتحاق بير امج التطوير المهني والتعليم المستمر داخل أو خارج العر اق، و المشاركة في المؤتمرات و الندوات و المحاضر ات، لما لها الأثر في رفع المستوى العلمي و المهني لهو لاء العاملين وتمكينهم من تقديم خدمة مميزة للمستفيدين.

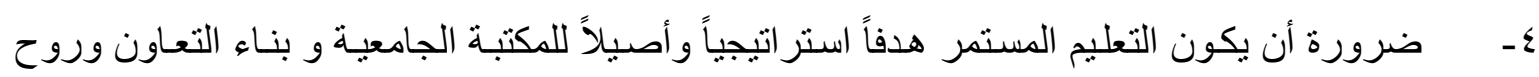
الجماعة بين العاملين لتحسين الأداء على جميع المستويات.

الموامش ومصادر البمث:-

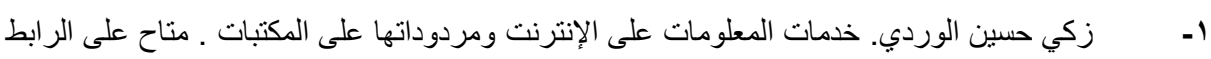

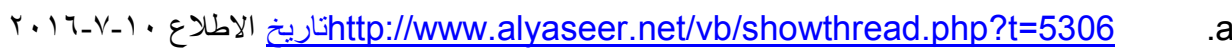

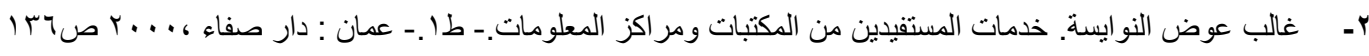

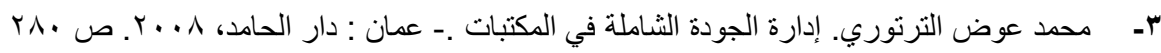

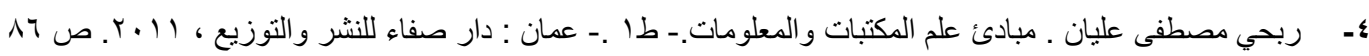

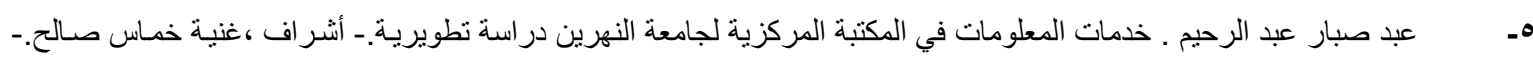

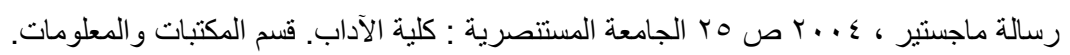

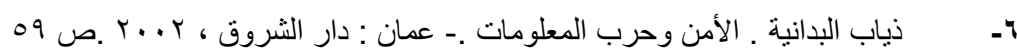

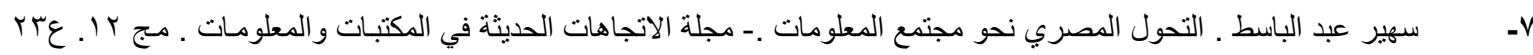

111 ص r...

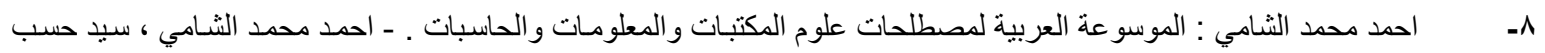

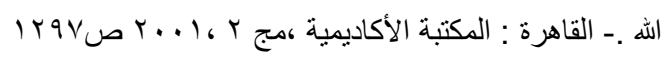

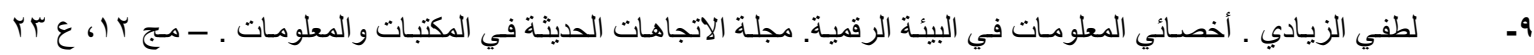

r. T... T.

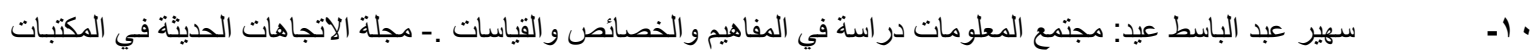

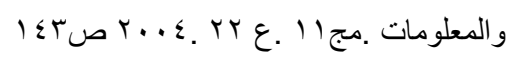

طارق محمود عباس ـ خدمات المكتبات الالكترونية نموذج للمكتبات الأمريكية ـ ـ مصر الجديدة : المركز الأصيل للطباعة،

$-11$

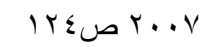

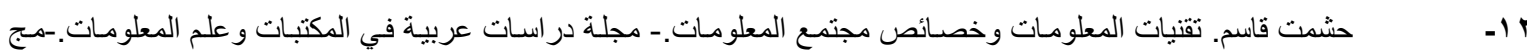

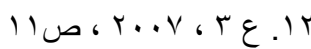

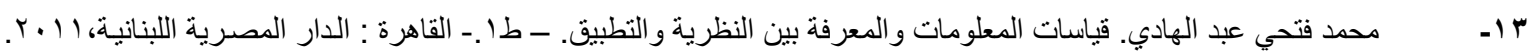
صن

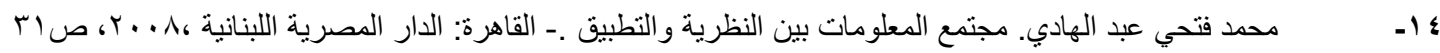

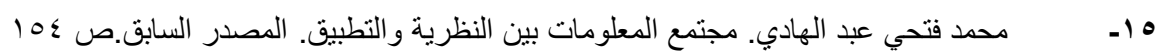

17

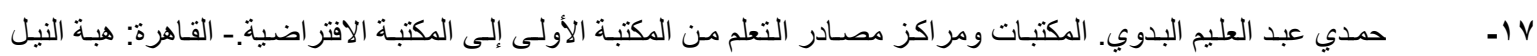

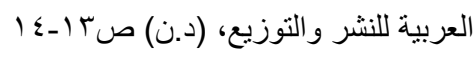

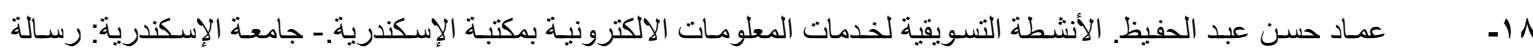

ماجستير، . 1.

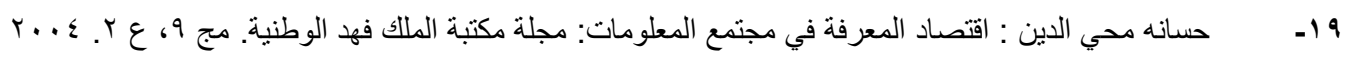

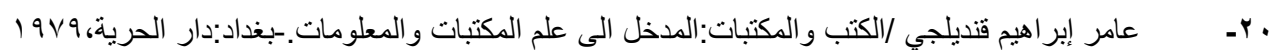

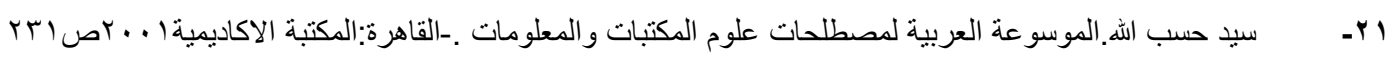

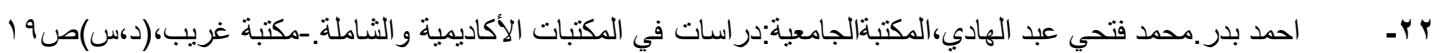




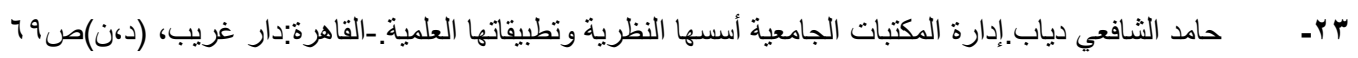

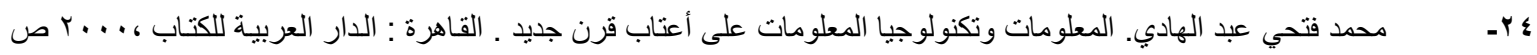
$\begin{array}{ll}1 \leqslant 0 & 1 \leqslant 0\end{array}$

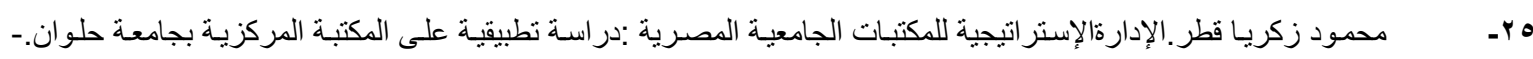

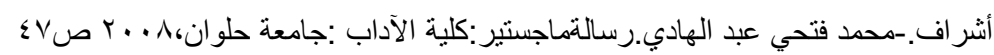

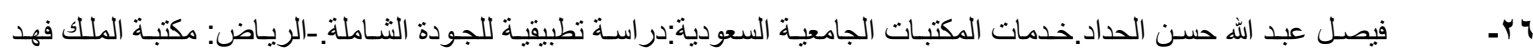

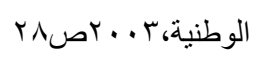

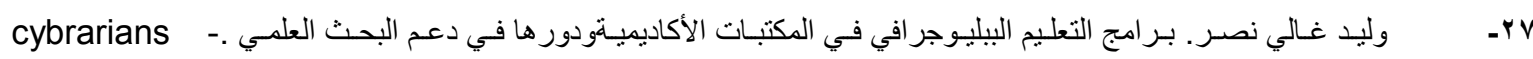

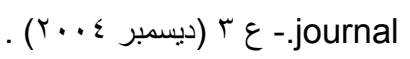

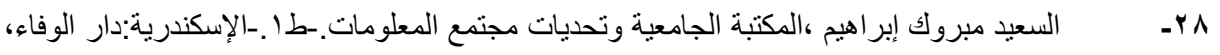

$$
11 \text { ll. }
$$

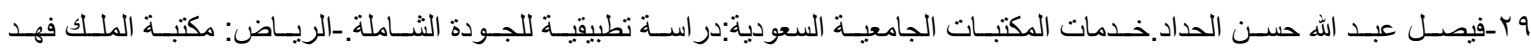

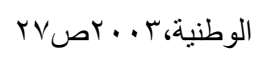

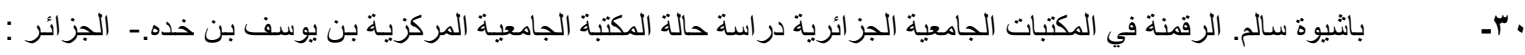

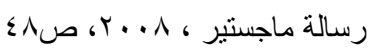

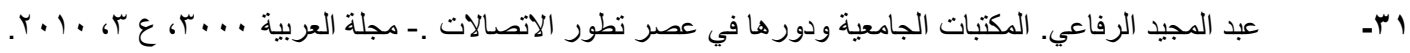

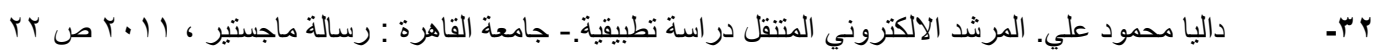

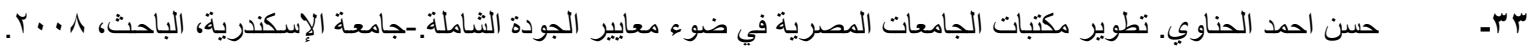

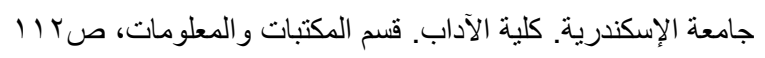

- Rubin, Rhea Joyce: Outcome measurement in academic, school and public libraries: a r selected bibliography and webography. 2009.

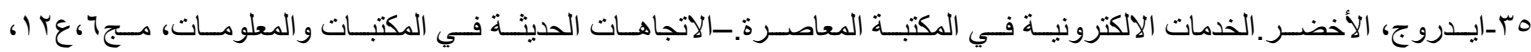

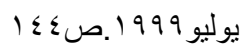

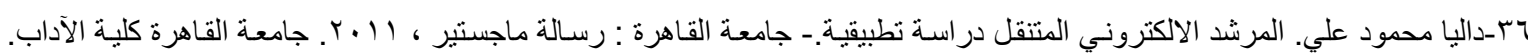
قسم المكتبات و المعلومات ص بكردي. V V-مخلص محمد خير اصطيف. المكتبات الجامعية في بيئة مجتمع المعرفة. كلية الآداب : جامعة حلوان : رسالة ماجستير ، 10 ـ با، ص ص

^ץ ـاحمد بدر .المكتبات الجامعية: تنظيمها و إدارتها وخدماتها ودورها في تطوير التعليم الجامعي و البحث العلىي .- دار غريب : القاهرة ، 0 ص. r...

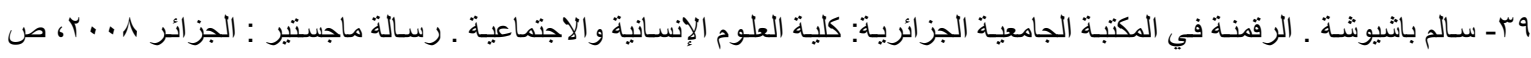
.101 • ـ ـاحمد حافظ إبر اهيم. خدمات المعلومات الالكترونية في المكتبة المركزية جامعة بغداد.- جامعة حلوان : رسالة ماجستير، ب ا ـ ب جامعة

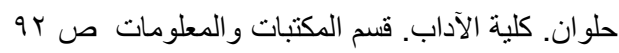

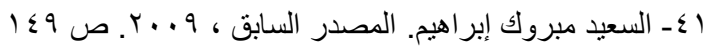

